\title{
To turn weakness into a strength-preoperative future liver remnant (FLR) augmentation with special focus on local tumor control and in-situ immunization for patients with advanced hepatocellular carcinoma (HCC) and liver cirrhosis (LC)
}

\author{
Oleksandr KORSHAK ${ }^{* 1}$, Olha SOKOLOVA ${ }^{2}$, Andrii ZHYLENKO', Denis FEDOROV ${ }^{1}$, Oleksandr HRINENKO', Oleksandr OSTAPYSHEN', \\ Maria STASSIUK ${ }^{3}$, Andriy HUSIEV', Oleksiy POPOV ${ }^{1}$, Alla KUBASHKO ${ }^{4}$, Vlad KROPELNYTSKYI ${ }^{4}$, Mykhailo KOSTYLEV ${ }^{3}$ \\ 'Department of Liver Surgery and Liver Transplantatione, National Institute of Surgery and Transplantology, Kyiv, Ukraine \\ ${ }^{2}$ Department of Oncology, National Institute of Surgery and Transplantology, Kyiv, Ukraine \\ ${ }^{3}$ Department of Radiology, National Institute of Surgery and Transplantology, Kyiv, Ukraine \\ ${ }^{4}$ Department of Pathology, National Institute of Surgery and Transplantology, Kyiv, Ukraine
}

Introduction: Portal vein embolization (PVE) provokes tumor progression. LC decreases FLR regeneration capacity doubling the risk for patient dropout. Our aim was to develop method of FLR augmentation that convert aggressive tumor into safe vaccine and prolonged waiting period for FLR regeneration into effective In situ immunization period.

Methods: 3 initially unresectable patients due to small FLR with advanced hepatocellular carcinoma (HCC) and LC were treated. Selective transarterial chemoembolization with short term biodegradable starch microspheres (DSM-TACE), into tumor bearing liver to be resected, was followed by simultaneous PVE of latter. Upon completion of PVE selective intratumoral immunotherapy (HIT-IT) with antiPD-L1 (atezolizumab 1,200 mg) into restored after DSM-TACE tumor arterial feeders (for selective connection with PD-L1 ligands located on tumor cells but not on normal human tissues) was done. DSM-TACE and HIT-IT was repeated one more time in all patients after postzenith decrease of T-cytotoxic cells.

Results: Predominantly T and NK cells response was observed. All patients had successfully underwent major liver resection upon sufficient FLR regeneration. In all 3 cases we had achieved effective local tumor control via total or subtotal HCC necrosis, even more, in $1(33 \%)$ case planned amount of liver resection was decreased due to achieved tumor downsizing. There were no severe morbidity or Immune-related adverse events (irAEs).

Conclusions: Herein we had proposed new aggressive but safe method of FLR augmentation for patients with HCC and LC that could potentially preclude drop out of patients during anticipated prolonged waiting period of FLR augmentation and possible improves long-term outcomes by means of tumor downsizing and HCC immunoscore conversion. 